\title{
La asistencia social en México. Una mirada desde el SNDIF***
}

\author{
Social assistance in Mexico. \\ A view from the SNDIF \\ A assistência social no México. \\ Uma visão do SNDIF
}

Recibido el 25 de junio de 2017. Aceptado el 23 de octubre de 2017

Elieth Blázquez Bonilla***
México

, Para citar este artículo:

Blázquez Bonilla, Elieth (diciembre, 2017). La asistencia social en México. Una mirada desde el SNDIF. Ánfora, 24(43), 189-212. Universidad Autónoma de Manizales. ISSN 0121-6538.

\section{Resumen}

Objetivos: establecer la importancia del programa de asistencia social SNDIF del Estado mexicano e identificar sus alcances y limitaciones en relación con el mejoramiento de la calidad de vida de la población. Metodología: estudio de Revisión que documentó e hizo análisis crítico de la información emitida por el Sistema Nacional para el Desarrollo Integral de la Familia (SNDIF). Se revisaron documentos primarios como la Consulta Nacional de Información Básica a los Sistemas Municipales 2015 (CNIBSM-DIF), así como informes, comunicados, libros, artículos y notas periodísticas.

\footnotetext{
* Este artículo se deriva de la investigación denominada "El Sistema Nacional para el Desarrollo Integral de la Familia (SNDIF) en el horizonte de la asistencia social en México. Antecedentes, presente y retos", presentada por la autora para optar por el título de Doctora en Administración Pública por el Instituto Nacional de Administración Pública, A.C., 2017. Enero 2015. Investigación en proceso.

** Sistema Nacional para el Desarrollo Integral de la Familia, el cual es una institución del Estado mexicano que funge como centro de asistencia social. Se fundó en 1977, tiene una estructura descentralizada, es decir, el Sistema Nacional desempeña el papel de coordinador y dirigente del trabajo realizado por los Sistemas Estatales y los Sistemas Municipales.

*** Maestra en Ciencia Política y Gestión Pública. Doctoranda en Administración Pública. Administrador Público. Profesora e investigadora de la Benemérita Universidad Autónoma de Puebla, México. Correo electrónico: eliethblazquezbonilla@gmail.com y eliethb1@hotmail.com
} 
Resultado: el SNDIF mantiene diversas estrategias de asistencia social, entre las que se destacan 1) la defensa de los derechos de la infancia, 2) personas con discapacidad, 3) la Cruzada contra el Hambre y 4) el apoyo a los migrantes. Si bien, el gobierno federal asignó para el 2017, \$1,357,015.61 pesos mexicanos al rubro de Protección social, se evidenció que sus acciones no lograron abarcar el total de la población necesitada. Conclusiones: la asistencia social en México es un proceso con dos caras: por un lado, es una obligación del Estado respecto al bienestar de la población; por otro, es un sistema de control y sometimiento de la gente. La asistencia social en el gobierno de Enrique Peña Nieto ha sido instrumento idóneo para estabilizar su poder y promover la continuidad de dicha elite dominante.

Palabras clave: Asistencia social; México; SNDIF.

\section{Abstract}

Objective: to establish the importance of Mexico's SNDIF social assistance program and to identify its scope and limitations regarding the improvement in the population's quality of life. Methodology: this review study documented and critically analyzed the information issued by the National System for the Integral Development of the Family (SNDIF). Primary documents such as the National Consultation on Basic Information for Municipal Systems 2015 (CNIBSM-DIF), as well as, reports, communiqués, books, articles and journalistic notes were reviewed. Result: the SNDIF maintains different social assistance strategies that include: 1) the advocacy for children's rights; 2) people with disabilities; 3) the Crusade against Hunger; and 4) support for migrants. Although the federal government allocated \$1, 357,015.61 Mexican pesos to Social Protection in 2017, it was noted that its actions failed to reach the total population in need. Conclusions: social assistance in Mexico is a two-sided process; on the one hand, it is a State obligation on the population's welfare, and on the other, it is a system of control and a subjugation of the people. Social assistance in the government of Enrique Peña Nieto has been an appropriate tool to balance its power and promote the continuity of such ruling elite.

Keywords: Social assistance; Mexico; SNDIF.

\section{Resumo}

Objetivo: estabelecer a importância do programa de assistência social SNDIF do Estado mexicano e identificar seus alcances e limitações em relação à melhoria da 
qualidade devidadapopulação. Metodologia:estudo de Revisãoquedocumentouefez análise crítica da informação emitida pelo Sistema Nacional para o Desenvolvimento Integral da Família (SNDIF). Foram revisados documentos primários, como a Consulta Nacional de Informação Básica aos sistemas Municipais de 2015 (CNIBSM-DIF), assim como informes, comunicados, livros, artigos e notícias. Resultado: o SNDIF mantém várias estratégias de assistência social, nas que se destacam 1) Defesa dos direitos da Criança, 2) Pessoas com Deficiência, 3) a Cruzada contra a Fome e 4) o apoio para os migrantes. Enquanto o governo federal destinou para 2017, \$1,357,015.61 pesos mexicanos para proteção social, mostrou-se que suas ações não conseguiram cobrir o total da população em necessidade. Conclusões: a assistência social no México é um processo com duas faces: de um lado, é uma obrigação do Estado em relação ao bem-estar da população; do outro, é um sistema de controle e subjugação do povo. A assistência social no governo de Enrique Peña Nieto tem sido um instrumento ideal para estabilizar seu poder e promover a continuidade de tal elite dominante.

Palavras-chave: Assistência social; México; SNDIF. 


\section{Introducción}

Asistir significa socorrer, favorecer en caso de necesidad. La ayuda es un ejercicio desligado de la idea de lucro, se asocia con fines de tipo humanitario y actos de solidaridad. Valckax (2007) señala que "la asistencia social puede entenderse como toda acción dirigida a la población que en diversos momentos de la historia ha sido marginada y/o excluida de las condiciones materiales necesarias para su reproducción" (p. 71). Fletes (2004), considera que la asistencia social "se trata del conjunto de disposiciones legales y acciones llevadas a cabo por las instancias gubernamentales en un plano federal, estatal y municipal, dirigidas a atender las necesidades básicas, pero también urgentes, de individuos y grupos de individuos que no están en condiciones de satisfacerlas por ellos mismos" (2). Y Ander-Egg (1995) concibe la asistencia social como el "conjunto de servicios prestados a aquella parte de la población que tiene problemas que no puede resolver por sí misma” (p. 7).

Las definiciones citadas plantean de manera concordante que la asistencia social consiste en acciones dirigidas a grupos vulnerables que necesitan ayuda para cubrir determinadas necesidades básicas. Como señala Fletes (2004), el concepto en sí mismo establece que "la asistencia no tiene un carácter preventivo" (p. 2), pues satisface "necesidades urgentes, se aboca a consecuencias y no a causas". Y agrega que "la asistencia social no es un mecanismo para superar la exclusión social, sino un punto de apoyo, puede verse como una estrategia de sobrevivencia" (p. 2).

La asistencia social se imparte, básicamente, por tres instituciones: la iglesia, el Estado y la sociedad civil. La impartida por la primera entidad se le denomina caridad, la segunda asistencia social y la tercera filantropía. Además de la denominación, una diferencia central en la acción de estos tres organismos consiste en que la asistencia social "es un mandato legal", es decir, el Estado como ente regulador tiene la obligación de resarcir las necesidades de la población que lo requiera. "La obligación del Estado mexicano de ejercer la asistencia social, se deriva de los artículos tercero y cuarto constitucional y más específicamente de la Ley General de Salud" (Fletes, 2004, p. 2).

Ahora, a pesar de que la asistencia social es una obligación del Estado, el apoyo es percibido por la población como una acción generosa de las autoridades y ello convierte la asistencia en un intercambio de ayuda por lealtad. Ramírez (2011) plantea que "en la relación de asistencia establecida entre el gobierno y los grupos beneficiarios lo recibido no es representado como un derecho ciudadano, sino como una ayuda; esto, $[\ldots]$ en lugar de fomentar la construcción 
de ciudadanía, genera formas de relación paternalistas, que entrañan gratitud y exponen a las poblaciones beneficiarias a prácticas clientelares” (p.137).

Este trabajo aborda de manera específica la función desempeñada por el Sistema Nacional para el Desarrollo Integral de la Familia (SNDIF) en el entramado de la asistencia social en México en el sexenio de Enrique Peña Nieto. A manera de contexto es importante señalar que el SNDIF se instituyó el 13 de enero de 1977, con la finalidad de que una institución organizara y dirigiera los diversos programas de asistencia social. El SNDIF se encuentra integrado por 32 sistemas estatales DIF y los sistemas municipales DIF (alrededor de 1,500 de los 2,414 municipios mexicanos). Siendo un organismo público, descentralizado, con personalidad jurídica y patrimonios propios (Talavera, 2004, p. 4).

El SNDIF implementa modelos de atención como una estrategia del gobierno federal con perspectiva familiar y comunitaria, a fin de promover el fortalecimiento de la familia, la niñez y el individuo en comunidad; además, brinda a sus integrantes nuevas formas para una mejor gestión a través de diversos procesos, relaciones intergubernamentales e interinstitucionales para fortalecer la capacidad de respuesta a la población vulnerable. A la vez, se incide en la manera de pensar del personal que se capacita en la búsqueda de certificaciones que emite el SNDIF; con ello, se vigoriza la entrega de los servicios de asistencia que brindan los Sistemas Estatales para el Desarrollo Integral de la Familia (SEDIF).

El SNDIF instaura Programas de atención a población vulnerable como una acción del gobierno federal a fin de promover el fortalecimiento de la familia y los individuos en estado de vulnerabilidad que representa un "factor de riesgo interno de un sujeto o sistema expuesto a una amenaza, correspondiente a su disposición intrínseca a ser afectado o de ser susceptible de sufrir daños" (Cardona, 2001, p. 11). El Sistema aplica 13,154 programas de asistencia social en 1,543 municipios.

Así pues, el SNDIF es una institución descentralizada que ramifica su poder y ejercicio a través de Sistemas Estatales (SEDIF) y Sistemas Municipales (SMDIF). Estas instancias son las unidades primarias de asistencia social gubernamental, es decir, son organismos que mantienen contacto directo con las demandas y necesidades de la población. La coordinación, organización y guía del SNDIF puede ser muy eficiente; pero para que dicha efectividad tenga un impacto real en la población es imprescindible el trabajo realizado por los sistemas estatales y los municipales. Las funciones desempeñadas por los SEDIF y los SMDIF son fundamentales, pues son el orden administrativo más próximo a la ciudadanía.

El SNDIF detenta una estructura organizada para gestionar recursos económicos que permiten la aplicación de los diversos programas. El mayor 
porcentaje de recursos provienen de la gestión que dicha institución lleva a cabo en cada municipio, es decir, el $76 \%$ de los recursos provienen del municipio, el $6 \%$ de cuotas de recuperación, el $4 \%$ recursos propios y el $3 \%$ de ingresos estatales y federales. Para que cada institución aplique los programas de acuerdo con las necesidades de cada región y tengan un mayor impacto, es clave la conformación de compendios y esquemas que regulen y sistematicen el trabajo de cada organismo, el uso de sus recursos y la aplicación de programas (Sistema Nacional para el Desarrollo Integral de la Familia [SNDIF], 2015).

El SMDIF cuenta con una plantilla laboral de 68,638 mujeres y hombres, la cual está contenida primordialmente en la zona centro y abarca el $31 \%$ de los trabajadores. Del total de los trabajadores, el 76\% está contratado por nómina, el $13 \%$ es personal sindicalizado, el $6 \%$ está conformado por voluntarios y el $5 \%$ se encuentra laborando bajo otro tipo de contratación (SNDIF, 2015). El voluntario es una figura central en el ámbito de la asistencia social y se refiere a "la persona que de manera libre presta sus servicios a terceros sin recibir por ello una compensación económica” (Serna, 2010, p. 44). El voluntariado representa fuerza de trabajo que no recibe ningún tipo de remuneración.

Además, el SMDIF cuenta con una infraestructura importante que le permite operar. De acuerdo con datos obtenidos en la Consulta Nacional de Información Básica a los Sistemas Municipales para el Desarrollo Integral de la Familia, 2015 (SNDIF, 2015), el 67.2\% cuenta al menos con un establecimiento de asistencia social. Dicho dato se recabó de 2,102 municipios que representan el $86 \%$ de los municipios totales existentes; entre ellos, poco menos de la mitad de los municipios del país cuentan con algún centro de atención pues se ha logrado institucionalizar dicho servicio generando ámbitos reales y materiales que proporcionan determinada ayuda.

Por su parte, el SNDIF tiene 13 tipos de instalaciones en las cuales se atiende a la población, como son casas cuna, casas hogar para niñas y niños, casas hogar para personas adultas mayores, casas de día o medio camino para personas adultas mayores, centros de atención infantil comunitario, centros de atención y desarrollo infantil, espacios de alimentación escolar, espacios de alimentación comunitaria, albergues para niñez migrante no acompañada, albergues para población vulnerable, unidades básicas de rehabilitación y consultorios médicos.

Los sistemas municipales tienen claramente definidos los sectores a los que destinan la asistencia. De acuerdo con los datos recolectados por la Consulta Nacional, el $27 \%$ de los programas elaborados tienen como objetivo atender aspectos relacionados con la comunidad, el 19\% consistió en programas dirigidos a niñas, niños y adolescentes de o a 18 años, el 9\% son programas de atención a la familia, $9 \%$ para adultos mayores, $7 \%$ población vulnerable, $6 \%$ para personas con discapacidad, $5 \%$ otros y $3 \%$ de los programas destinados a adolescentes y 
mujeres. Este tipo de organización es óptima, así como también, la segmentación de los sujetos destinatarios de la asistencia social. Como señalan García y Salazar (2013) "la vulnerabilidad se presenta en el interior y en el exterior de las familias, y por lo tanto, son necesarios los cambios materiales y simbólicos. [Es importante el] cumplimiento de los derechos en la familia (salud, educación, trabajo, apoyo social o comunitario, asociatividad, confianza)” (p. 45).

Este artículo tiene como objetivo revisar el impacto que han tenido los programas de asistencia social impartidos por el Estado mexicano contemporáneo, centrándose en el papel desempeñado por el Sistema Nacional para el Desarrollo Integral de la Familia (SNDIF). El paso de la autora de este trabajo como directora general del Sistema para el Desarrollo Integral de la Familia en el municipio de Puebla durante el periodo 2005- 2008, suscitó preguntas como ¿Cuáles son los alcances que han tenido los programas impulsados por el Sistema Nacional para el Desarrollo Integral de la Familia en el sexenio de Enrique Peña Nieto? Y ¿Cuáles son sus limitaciones? Con ello, se evidenció la importancia que tiene evaluar los programas de asistencia social impulsados por dicha instancia para mejorar la calidad de vida de la población vulnerable. Y otra razón para este estudio es que, por lo general, las valoraciones no se llevan a cabo con objetividad al interior de estas instituciones, lo cual impide que los representantes vean de manera crítica la repercusión de los programas para redirigir o reestructurar las acciones emprendidas.

\section{Metodología}

La presente investigación está sustentada en el método cualitativo. Para lograr el objetivo de revisar el impacto del SNDIF en el mejoramiento de la calidad de vida de la población que se atiende se aplicó una Revisión de la gestión documental a través de los informes, comunicados, discursos, cuestionarios como la Consulta Nacional de Información Básica a los Sistemas Municipales (CNIBSM-DIF), libros, artículos y notas periodísticas. Se dio una importancia primordial a la revisión de documentos emitidos por el Sistema Nacional para el Desarrollo Integral de la Familia (SNDIF), pues estos representan el discurso oficial de las acciones que lleva a cabo el Estado para asistir a la población vulnerable.

A través de la revisión documental se recabaron datos sobre la asistencia social en México, y de manera más específica sobre el SNDIF. En un primer momento se desglosan las promesas de campaña de Enrique Peña Nieto y las acciones que emprendió al obtener la presidencia en 2012, referentes a la asistencia social. 
Posteriormente, se desarrollan los aspectos legales que exhortan al Estado a implementar políticas de asistencia social. Finalmente, se explican los acciones emprendidas por el SNDIF, retomando cuatro programas centrales: 1) Defensa de los derechos de la infancia, 2) Apoyo a personas con discapacidad, 3) Cruzada contra el hambre y 4) Apoyo a los migrantes. Sobre cada uno de los programas se ofrecen datos e se identifican los alcances y las limitaciones de estos.

La recolección de datos y su análisis fueron procesos sistemáticos y ordenados, desarrollados en las siguientes fases: 1) obtención de información, 2) Captura, transcripción y organización, 3) Codificación y 4) integración (Fernández, 2006). Cabe profundizar que en la fase tres, codificación de la información, se llevó a cabo una clasificación de ideas, conceptos y temas comunes. Se clasificaron datos estadísticos, programas aplicados, datos históricos y fundamentos teóricos para comprender y analizar el ejercicio de la asistencia social en el México contemporáneo.

\section{Resultados}

El 30 de marzo de 2012, Enrique Peña Nieto inició su campaña en Guadalajara, y como proceso inaugural, firmó cinco compromisos "para cambiar a México". De manera breve, estos cinco compromisos consistían en "recuperar la paz, combatir la pobreza, impulsar la educación, generar empleos y recuperar el liderazgo en el mundo" (PRI, 2012; Animal Político, 2012, Radio Fórmula, 2012). Ya consolidado como presidente de la república, Peña Nieto "inició una cruzada en pro del desarrollo social", planteando tres programas: "Cruzada Nacional contra el Hambre, Seguro de vida para las Jefas de Familia y Programa 65 y más" (Cordero, 2012). Posteriormente, en la Agenda por México firmada por Enrique Peña Nieto y el Consejo Coordinador Empresarial en diciembre de 2012, se encontró una vinculación entre las instituciones gubernamentales y el sector privado para atenuar la pobreza y precariedad del país.

El 2 de septiembre de 2004, en el Diario Oficial de la Federación salió publicada la Ley de asistencia social, la cual fue reformada en 2014, estableciendo en el Artículo 3 que

Se entiende por asistencia social el conjunto de acciones tendientes a modificar y mejorar las circunstancias de carácter social que impidan el desarrollo integral del individuo, así como la protección física, mental y social de personas en estado de 
necesidad, indefensión desventaja física y mental, hasta lograr su incorporación a una vida plena y productiva.

La asistencia social comprende acciones de promoción, previsión, prevención, protección y rehabilitación.

En dicha ley, se ratifica que el Estado fungirá como rector de la asistencia social tanto pública como privada. El Estado coordinará y supervisará el trabajo realizado por el Sistema Nacional de Asistencia Social Pública y Privada (Ley de asistencia social, 2014), enfocándose principalmente en fomentar el desarrollo integral de la familia (Artículo 5). Esta ley fue ratificada por el gobierno de Enrique Peña Nieto, modificando que dentro de los Sujetos de asistencia social se deben contemplar niños migrantes y repatriados, niños víctimas de conflictos armados y de persecución étnica o religiosa y huérfanos. De igual manera, se estableció que las mujeres que están en periodo de gestación y madres adolescentes son sujetos que deben ser asistidos socialmente (Artículo 4) (Ley de asistencia social, 2014).

En el 4to Informe de Gobierno, el presidente Enrique Peña Nieto planteó que la política de desarrollo social es un elemento central en su sexenio, pues a través de ésta, es posible generar condiciones de “inclusión y bienestar entre los grupos más vulnerables de la población” (2016, p. 183). Para alcanzar estos objetivos, estableció la Estrategia Nacional de Inclusión, en la que participan el gobierno, el sector empresarial y organizaciones de la sociedad civil. El programa primordial ha sido la Cruzada Nacional contra el Hambre, que está coordinada principalmente por la Secretaria de Desarrollo Social (SEDESOL), pero en la cual, el SNDIF trabaja a partir de su perspectiva asistencial, sus funciones y con los grupos vulnerables que dicha institución atiende.

De acuerdo con la página Transparencia presupuestaria del gobierno mexicano (Gobierno Federal, 2017), el gobierno federal asignó para el 2017 \$1,357,015.61 millones de pesos mexicanos al rubro de Protección social, distribuido de la siguiente manera: $\$ 1,074,926.22$ millones de pesos, lo cual equivale al $79.21 \%$, se asignó a planes de Edad avanzada; \$128,589.86 millones de pesos (9.48\%) a Otros de Seguridad Social y Asistencia Social; $\$ 115,294.08$ millones de pesos (8.80\%) a Otros grupos vulnerables; $\$ 19,186.98$ millones de pesos (1.41\%) a Enfermedad e incapacidad; \$12,354.35 millones de pesos (0.91\%) a Familia e hijos y $\$ 868.77$ millones de pesos (0.06\%) a Apoyo social para vivienda.

Estos datos muestran un aumento en comparación con los recursos asignados en 2016. De acuerdo con el Presupuesto de Egresos de la Federación 2016, se asignaron $\$ 738,340.0$ millones de pesos al rubro de Protección Social, dinero que se distribuyó en los siguientes programas: PROSPERA (\$81,689.9 millones de 
pesos); Pensión para Adultos Mayores (\$39,486.5 millones de pesos); Programa de Infraestructura Indígena ( $\$ 7,590.7$ millones de pesos) y Estancias Infantiles (\$4,149.8 millones de pesos).

El SNDIF trabaja en diversas actividades de asistencia social, sin embargo, en este análisis se retomaron cuatro ámbitos primordiales en la labor de dicha institución: 1) la defensa de los derechos de la infancia; 2) personas con discapacidad; 3) la Cruzada contra el Hambre y 4) el apoyo a los migrantes.

La defensa de los derechos de la infancia es una labor que está fundamentada en la Constitución Política de los Estados Unidos Mexicanos, la cual en el artículo $4^{\circ}$ señala que

El Estado, en todas las decisiones y actuaciones, velará y cumplirá con el principio del interés superior de la niñez, garantizando de manera plena sus derechos a la educación y sano esparcimiento para su desarrollo integral, principio que deberá guiar el diseño, ejecución, seguimiento y evaluación de las políticas públicas dirigidas a la niñez (Diario Oficial, 30 de abril, 2014).

Para trabajar en este ámbito, el Gobierno de la República abrió un programa de becas otorgadas a niños y adolescentes en condición de vulnerabilidad. De acuerdo con datos emitidos por el gobierno "durante el ciclo escolar 2015-2016 se otorgaron becas a más de 7.7 millones de alumnos de escuelas públicas del país. De esta manera, se benefició a casi tres de cada 10 estudiantes (29.9\%) en los distintos niveles de estudio. En educación básica se distribuyeron 5.1 millones de becas, en media superior 1.9 millones y en educación superior 761.7 miles de estos apoyos" (Peña, 2016b, p. 374).

El programa de becas está coordinado por la Secretaría de Educación Pública (SEP) y por las instituciones educativas y contó en 2016 con un presupuesto de 12,651.8 millones de pesos (Peña, 2016b, p. 374). El SNDIF como institución de asistencia social apoyó dicha iniciativa con actividades complementarías como la entrega de mochilas y útiles escolares (SNDIF, 2013). El interés del gobierno por reafirmar dicho compromiso se ratificó con el convenio firmado por el Gobierno de la República y el Fondo de las Naciones Unidas para la Infancia (Unicef), en el que las partes se comprometieron a llevar a cabo un trabajo colaborativo para lograr el cumplimiento de los derechos de las niñas y los niños (SNDIF, 2013).

El programa de becas aborda un aspecto de instrucción y formación de los niños que, sin lugar a dudas, es parte importante del bienestar de los niños; sin embargo, el papel del SNDIF en este tema, tiene un aspecto más global, enfocado principalmente a generar buen calidad de vida para las niñas y niños de sectores vulnerables. Es por eso, que parte de sus actividades consisten en darle albergue 
y cuidado a niños que viven en indefensión, señalando que estas casas cuna buscan "otorgar sólidas herramientas para que en un futuro sean ciudadanos de bien" (SNDIF, 2013).

Por ejemplo, de acuerdo con datos emitidos por el SNDIF, la casa hogar para niños ‘Graciela Zubirán’ “alberga a 197 niñas y 122 niños que tienen entre 4 meses y 20 años”, en la cual reciben “atención médica, jurídica-social, psicológica, vestido, alimentación, educación, actividades recreativas y culturales" (SNDIF, 2013). Entre las actividades recreativas, el SNDIF celebra el día del niño, realiza posadas y lleva a los niños a funciones de cine gratuitas, esto último apoyándose con empresas privadas como fue el caso de Cinépolis (SNDIF, 2013/2014; Gobierno de la República, 2014).

El trabajo que se realizó en las casas cuna del DIF es adecuado para generar mejor calidad de vida de las niñas y los niños. Si se piensa en la Pirámide de las necesidades de Maslow se puede observar que la atención que reciben en estos centros cubren las etapas de dicha pirámide; es decir, las actividades realizadas y los servicios recibidos satisfacen en los niños sus necesidades fisiológicas, de seguridad, afiliación, reconocimiento y autorrealización. Los niños y niñas de los albergues son visualizados como seres holísticos en los que se deben cubrir tanto necesidades básicas como aspectos de entretenimiento y desarrollo personal.

Sin embargo, este trabajo es reducido en comparación con las necesidades y demandas de la infancia mexicana. Los datos ofrecidos por instituciones internacionales evidencian que el trabajo realizado por el SNDIF es escaso y está muy alejado de instaurar un país que garantice seguridad para los niños y las niñas. La organización internacional Save the Children señala que "la niñez es una etapa de la vida en que los niños y las niñas puedan crecer, aprender y jugar en un entorno seguro. [...] Sin embargo, por lo menos un 25\% de los niños y las niñas del mundo no goza de estos derechos" (2017). Este organismo creo el Índice de Peligros para la Niñez, instrumento con el que comparó a 172 países, asignando el primer lugar al país en donde la niñez enfrenta menos peligros. México ocupa el lugar 90 en dicho ranking con los siguientes índices: presenta un 13,2\% en tasa de mortalidad de niños y niñas menores de 5 años, índice medio que se incrementa de manera marcada en estados como Chiapas $(18,5)$, Oaxaca (18), Campeche (17,8), Guerrero (17,7) o Puebla (17,2).

El 12,4\% presenta retraso en el crecimiento, "esto quiere decir que poco más de millón y medio de niñas y niños no lograrán alcanzar su máximo potencial debido al hambre, [siendo más propensos] los niños y niñas residentes de zonas rurales, provenientes de los hogares más pobres y de origen indígena” (Save de Children, 2017, p, 4). El 15,2\% de niños y niñas en edad de educación primaria y secundaria no van a la escuela; el 10,4\% trabaja para ayudar económicamente a su familia. El 15,4\% de adolescentes están casados o viven en pareja; el 62,2\% de 
adolescentes entre 15 y 19 años tienen un hijo o una hija; el o,1\% de los niños y niñas fueron desplazados por la fuerza debido a un conflicto y el 5,5\% de niños y niñas han sido asesinados (Save de Children, 2017).

Como se señaló anteriormente, un segundo sector al cual va dirigido el trabajo del SNDIF, son las personas con discapacidad. Se debe anotar que el 13 de diciembre de 2006 la Asamblea General de las Naciones Unidas aprobó la Convención Internacional sobre los Derechos de las Personas con Discapacidad, la cual quedó abierta a firma el 30 de marzo de 2007, fecha en la que México y otros 69 países más firmaron y ratificaron dicho convenio (ONU, 2006). Este documento busca reafirmar que todas las personas con o sin discapacidad tienen derecho a una vida plena, a ejercer sus derechos sociales y políticos, a tener acceso a salud, educación, tiempo de ocio y trabajo, reconociendo que

La discapacidad es un concepto que evoluciona y que resulta de la interacción entre las personas con deficiencias y las barreras debidas a la actitud y al entorno que evitan su participación plena y efectiva en la sociedad, en igualdad de condiciones con las demás (Convención Internacional sobre los Derechos de las Personas con Discapacidad, 2006).

Es decir, como señala la organización civil Discapacidad y Derechos en este aparte de uno de sus videos:

[Las personas] no [tienen] discapacidad sino que la discapacidad aparece cuando [dejan] de estar en igualdad de oportunidades con el otro.

Lo que tienen las personas son algunas limitaciones que pueden sobreponerse gracias a ciertos ajustes que implementados garantizan la igualdad de oportunidades.

Son la sociedad y el gobierno los que tienen que garantizar estos ajustes. Si no los garantizan, estaríamos aceptando discriminaciones y abusos que a estas alturas no debemos permitir ${ }^{1}$.

Con base en estos compromisos, el SNDIF lleva a cabo diversas acciones que buscan consolidar una sociedad incluyente. La labor aborda cuatro esferas: reconocimiento como seres integrales, alimentación, salud y autorrealización. Sobre el reconocimiento, el trabajo del SNDIF parte de la premisa de que "la

1. Video completo en https://www.youtube.com/watch?v=1gg097y5bHw 
inclusión de las personas con discapacidad en todos los ámbitos no es una dádiva, sino un derecho inherente a cada uno de los seres humanos" (SNDIF, 2012).

En cuanto a la alimentación, la institución ha entregado despensas y apoyos a personas con discapacidad (SNDIF, 2013). En salud, el organismo entregó 125 camionetas que facilitan el traslado de las personas, así como también, sillas de ruedas, prótesis y andaderas (SNDIF, 2016/ 2013). En el tema de la autorrealización, dicho organismo realizó en 2013 los XL Juegos Nacionales deportivos sobre silla de ruedas y en 2015, Enrique Peña Nieto entregó la Norma Mexicana en Igualdad laboral y No discriminación, la cual "establece que empresas e instituciones incluyan en su fuerza laboral a personas con discapacidad” (SNDIF, 2013/2015).

De acuerdo con datos emitidos por dicha institución, ésta "cuenta con 104 centros y mil 444 unidades básicas de rehabilitación”, como el Centro de Rehabilitación Gaby Brimmer, el Centro Nacional de Atención a Personas Ciegas y Débiles Visuales y el Centro Municipal de Equinoterapia y Rehabilitación Integral (MERI), entre otras. En 2012 atendieron a

902 mil 247 personas, se otorgaron 202 mil 824 consultas médicas; se proporcionaron 120 mil 78 consultas en el área de psicología, pedagogía, estudios de gabinete, trabajo social y optometría. Se brindaron un millón 749 mil 849 sesiones de terapia física, ocupacional y de lenguaje [...] en materia de inclusión de personas con discapacidad en los ámbitos educativos, laboral y social fueron incluidas tres mil 491 personas (SNDIF, 2012/2013).

El trabajo realizado por el SNDIF en este ámbito ha sido importante, sobre todo porque va dirigido a uno de los sectores más vulnerable de la población. Sin embargo, hay que señalar que al igual que los otros programas, el apoyo y la asistencia social que se otorga es reducido en comparación con las necesidades reales existentes. De acuerdo con datos emitidos por el Instituto Nacional de Estadística y Geografía (INEGI, 2017), hay en México 498,640 personas con limitación en la actividad para escuchar; 401,534 personas con limitaciones en la actividad para hablar o comunicarse; 2,437,397 personas con limitación para caminar o moverse y 1,292,201 personas con limitación para ver. Esto da un total de 4,629,772 personas potenciales de requerir alguno de los apoyos ofrecidos por el SNDIF, dato que evidencia lo rebasados que están los servicios de asistencia social gubernamental.

El tercer programa que se aborda en este trabajo es la Cruzada Nacional contra el Hambre $(\mathrm{CNcH})$, el cual está constituido por 90 programas referentes a necesidades alimentarias, de salud, educación, mejoramiento y servicios en mil 12 municipios del país. El proyecto busca empoderar a la población, organizándola 
para que cada una trabaje en el desarrollo y progreso de sus comunidades. Dicho plan está constituido por asambleas y comités conformados por la población, miembros de la sociedad civil y jóvenes universitarios (Gobierno Federal, 2016; Secretaría de Gobernación, 2016).

El Gobierno de la República ha señalado que el programa Cruzada Nacional contra el Hambre ha tenido un impacto importante en la calidad de vida de las comunidades marginadas contempladas por dicho programa. Asevera que en junio de 2016 existían “12,987 comedores comunitarios en operación que benefician a 1,119,00 personas”, el Programa de Abasto Rural ha atendido a “22,086 localidades a través de 24,289 tiendas fijas, y a 2,858 localidades con unidades móviles". "Se cuenta con 12,722 huertos familiares [...], el Programa Pensión para Adultos Mayores benefició a 3.8 millones de personas” (Gobierno federal, 2014).

De igual manera, a través de las "estancias afiliadas al Programa de Estancias Infantiles para Apoyo a Madres Trabajadoras, se atendió a 741,847 niñas y niños que recibieron tres alimentos al día"; así como, la entrega de "suplementos alimenticios a 973,721 niñas y niños de seis a 59 meses de edad”. Y se apoyaron “7,619 espacios alimentarios (cocinas, desayunadores o comedores escolares o comunitarios), que mejoraron la situación de 685,645 personas” (Peña, 2016a: 184).

En la Cruzada Nacional contra el Hambre, el SNDIF participa de manera específica, a cargo del programa Estrategia Integral de Asistencia Social Alimentaria (EIASA). Dicha estrategia se conformó en 2001, con la finalidad de coordinar todos los programas de asistencia alimentaria que emprendía cada DIF estatal, pues la descentralización de sus funciones en cada región requirió de una política que regulara y organizara las actividades emprendidas por cada Estado. La EIASA consiste en

Una nueva estructura de cuatro programas con actividades de focalización, orientación alimentaria y aseguramiento de la calidad. El principal objetivo de la Estrategia fue, hasta 2007, el de contribuir a mejorar el estado de nutrición de los sujetos de asistencia social el cual ha evolucionado de acuerdo al contexto actual de salud y nutrición (SNDIF, 2016, p. 6).

La EIASA está constituida por cuatro programas: 1) Desayunos escolares; 2) Atención alimentaria a menores de 5 años en riesgo, no escolarizados; 3) Asistencia alimentaria a sujetos vulnerables y 4) Asistencia alimentaria a familias en desamparo. 
De acuerdo con los resultados emitidos por el Gobierno Federal, estos programas han logrado mejorar la calidad de vida de la población más vulnerable. Durante el periodo de 2013-2016,

El Programa Desayunos Escolares distribuyó 907,452,236 desayunos diarios, en beneficio de 5,595,662 niñas y niños, en promedio al día, en 2,293 municipios. El Programa Atención a Menores de Cinco Años en Riesgo no Escolarizados abasteció 79,957,477 raciones alimentarias a 411,565 niñas y niños, en 1,692 municipios. El Programa Asistencia Alimentaria a Familias en Desamparo otorgó 1,310,799 despensas a 158,432 familias en 337 municipios. El Programa Asistencia Alimentaria a Sujetos Vulnerables repartió 5,745,431 despensas en beneficio de 910,729 personas en 2,015 municipios (2016, p. 184).

La discusión sobre la pertinencia de estos programas va más allá de los alcances que han tenido y de la existencia de sectores de la población que aún no cuenta con dichas prestaciones. El problema de los programas para disminuir los índices de pobreza es más profundo y difícil que las acciones inmediatas que se ejercen. La dificultad - como señala Susana Murillo- radica en que "la pobreza es inherente a la lógica del capital”, lo que está de fondo es la "desigualdad intrínseca a las relaciones sociales capitalistas” (Murillo, 2007). Erradicar la pobreza, implica necesariamente una modificación en las relaciones sociales de producción. De tal manera que estos programas ofrecen alimento a algunos sectores de la población, pero esto no elimina la pobreza existente en el país. De acuerdo con datos emitidos por el Consejo Nacional de Evaluación de la Política de Desarrollo Social (CONEVAL, 2017) en 2016 México contaba aún con 53.4 millones de personas en situación de pobreza.

Finalmente, se aborda el trabajo realizado por el SNDIF para apoyar a los migrantes en su tránsito por México. El fenómeno de la migración es un tema espinoso y complejo tanto en la aplicación de políticas públicas como en el ámbito de la asistencia social. Es posible observar que la asistencia social -en su transcurso histórico- ha sido una práctica ligada a la concepción de ciudadanía; es decir, en el país, los que eran concebidos como sujetos destinatarios para recibir apoyo social son aquellos que legalmente están concebidos como ciudadanos.

La asistencia social a migrantes es una acción que rompe con dicha concepción tradicional, pues implica el apoyo a los no ciudadanos, a los ilegales. Estas acciones son respuesta a una demanda global, de anteponer los derechos humanos sobre los derechos ciudadanos, aspecto que tiene legalidad en la Declaración Universal de los Derechos Humanos. 
La labor del SNDIF en este ámbito consiste en la instauración de albergues que ofrecen un espacio propicio a niños y niñas no acompañados y a mujeres migrantes. Estos albergues temporales son "espacios dignos para brindar la asistencia que requiere la población migrante, sobre todo a niñas y niños. [... Se brinda estancia cómoda, se propicia estabilidad física y emocional, [...] se ofrece asistencia humanitaria integral. [...] Los servicios son de atención médica, psicológica y alimentaría; cuenta con sala de cómputo, dormitorio, estancia de descanso, juegos infantiles, baños y lavaderos" (SNDIF, 2013).

De acuerdo con datos emitidos por el SNDIF (2013) "de enero a agosto de 2013, el DIF Nacional atendió 12 mil 881 niñas, niños y adolescentes no acompañados y repatriados". Dichas acciones y la continuación de estas fueron refrendadas por la titular del SNDIF, Laura Vargas, el 28 de agosto de 2013 en una reunión de trabajo de varios países realizada en Guatemala.

En contraposición a estos datos, investigadores de El Colegio de la Frontera Norte (París, et al., 2015) con apoyo de Ford Foundation, elaboraron un análisis de las políticas migratorias aplicadas en México. En dicho informe, los investigadores aseveraron que el SNDIF no cuenta con los recursos económicos y humanos necesarios para satisfacer las demandas y necesidades de los transmigrantes. En dicho documento plantean que

La falta de recursos humanos es aún más patente en el Desarrollo Integral de la Familia (DIF), donde faltan abogados, trabajadores sociales y psicólogos para proteger los derechos de menores migrantes, [...] los albergues de los DIF estatales son generalmente lugares muy pobres, sin infraestructura ni materiales (París, et al., 2015, p. 58).

La participación del SNDIF en la asistencia social a migrantes en México, es una labor en la que aún faltan más acciones. Sin lugar a dudas, este sector es de los más vulnerables, pues además de vivir en precariedad, viven en condiciones de exclusión que su misma ilegalidad les condiciona. Salvaguardar la vida de las niñas, niños y adolescentes migrantes es una estrategia en la que debe trabajar el gobierno actual y los subsecuentes. 


\section{Conclusiones}

La asistencia social en México es un proceso con dos caras: por un lado, es una obligación del Estado con respecto al bienestar de la población; por otro, es un sistema de control y sometimiento de la gente. La implementación de programas de asistencia social por parte del Estado mexicano cumple un papel imprescindible en la consolidación de hegemonía. Dichos programas asistenciales son un engranaje más en la compleja maquinaria capitalista, cumplen una función reproductora de las estructuras.

Las acciones realizadas son actividades que, sin lugar a dudas, resarcen y cubren ciertas necesidades de algunos sectores de la población; pero no son las acciones necesarias para disminuir la desigualdad y la pobreza. Esto, porque el sistema dominante requiere dar un poco y a ciertos sectores para mantener su legitimidad. Es necesario que la elite sea concebida por la población como sus dirigentes y representantes (Gramsci, 1999), pues como señaló Jean-Jaques Rousseau "el gobernante, a diferencia del padre y del amo, tiene necesidad de que su autoridad sea aceptada para que sea considerada legítima” (Bobbio y Bovero, 1986, p. 88).

Los programas de asistencia social desempeñan un papel clave en la consolidación de legitimidad. Es por eso, que todas las campañas electorales contienen como eje rector aquellas acciones que los gobernantes llevarán a cabo para lograr el bienestar de la población y el progreso del país. El gobierno de Enrique Peña Nieto tomó el tema de la pobreza como la base de sus propuestas de asistencia social, con tres puntos centrales: erradicar el hambre, crear el sistema de seguridad social universal y un sistema educativo. Los programas de asistencia social han sido los instrumentos idóneos para estabilizar su poder y promover la continuidad de dicha elite dominante.

La asistencia social es una acción necesaria social y políticamente. Aminora la precariedad en la vida de ciertos sectores, pero mantiene la estructura y su reproducción. La pertinencia de estos programas radica en la existencia de un sector de la población a la que satisfacen de manera somera, pero que sin lugar a dudas lo requieren y a pesar de que es una obligación del Estado, lo agradecen.

Como se desarrolló en el documento, el SNDIF delega a los Estados y municipios la ejecución de los programas. A pesar de esta descentralización y búsqueda por llegar a las regiones más recónditas, uno de los problemas del sistema es que existe una concentración de programas en determinadas regiones, pues mientras que en algunos municipios se aplican entre 1 y 5 , en otras zonas se llevan a cabo entre 11 y 15 . Este es un problema importante para una institución 
que creó una estructura con miras a llegar a las zonas más vulnerables que requieren algún tipo de intervención y asistencia del Estado.

El trabajo del SNDIF debe ir encaminado a ampliar el número de beneficiarios de dichos programas. Disminuir los índices de la tasa de mortalidad infantil (14.3 muertes por cada mil nacidos vivos de 2011-2013), aumentar la asistencia de los niños a la escuela (en 2015, 3.5\% de la población de 6 a 14 años no asistía a la escuela), reducir el trabajo infantil (en 2013, 2.5 millones de niñas, niños y adolescentes realizaban alguna actividad económica) (Instituto Nacional de Estadística y Geografía [INEGI], 2016) y aminorar la incorporación de niños y adolescentes al crimen organizado. Este último aspecto es parte de la violencia, abandono y negligencia del Estado, pues como señala José Luis Cisneros (2014, p. 13) "son niños y jóvenes abandonados por el Estado y condenados a sobrevivir en los márgenes de la ilegalidad, que les ofrece lo que las instituciones del Estado les han negado”.

Es importante que el SNDIF materialice en acciones las premisas que lo conforman. Dicha instancia establece que la asistencia social debe alejarse de construir una política social de beneficencia donde se tienen sólo beneficiados. A diferencia de esto, debe trabajar en consolidar ciudadanos promotores de su propio desarrollo, en el cual la profesionalización promueva la atención de las causas y no de los efectos. La prevención, la corresponsabilidad y la profesionalización de los servicios en asistencia social son los que en su conjunto pueden procurar el fortalecimiento de la sociedad (SNDIF, 2005).

La prevención debe estar encaminada a detectar los factores de riesgo de vulnerabilidad y establecer programas, proyectos, servicios y acciones encaminados a disminuirlos. Se trata de actuar antes de que se potencialicen las causales de la vulnerabilidad de manera que, en la medida de lo posible, la atención sea la última alternativa (SNDIF, 2005).

La corresponsabilidad se puede definir en dos aspectos: el primero, relacionado directamente con el sujeto de atención; en este sentido, el sujeto debe contribuir de manera activa en su propia transición y reincorporación social. Dejar de ser pasivo, un simple receptor de atención asistencial y convertirse en protagonista, de manera que participe y defina su propio empoderamiento. El segundo aspecto tiene que ver con otros actores de la asistencia social; instancias o instituciones que se corresponsabilizan del gran reto de su quehacer. Ante la gran demanda y las dificultades que representa asumir la tarea asistencial, la integración de distintos frentes permite una mejor coordinación y una multiplicación de la cobertura y la calidad de los servicios (SNDIF, 2005).

Finalmente, la profesionalización de los servicios debe estar dirigida a recuperar experiencias y producir conocimiento; a formar y capacitar en todos los niveles y ámbitos de acción para establecer parámetros mínimos de calidad 
y una operación planificada. Establecer normas mínimas de operación para los servicios asistenciales, las cuales permitan concretar una mejor planeación, construcción de programas y modelos de intervención (SNDIF, 2005).

La responsabilidad del Estado es llevar a cabo acciones que fortalezcan las instituciones existentes y desarrollar estrategias administrativas que propicien la eficiencia de su labor, amplíen su propuesta y que tengan un impacto trascendental en la vida de la población más necesitada.

\section{Referencias}

Ander E. (1995). Diccionario del Trabajador Social. México: Lumen.

Animal Político. (2012). Peña Nieto arranca campaña con "tres compromisos" y declaración patrimonial. Animal político. Recuperado de http://www. animalpolitico.com/2012/03/pena-nieto-arranca-campana-con-trescompromisos-y-declaracion-patrimonial/

Bobbio, N. y Bovero, M. (1986). Sociedad y Estado en la filosofía política moderna. México: Fondo de Cultura Económica.

Cardona, O. (2001). La necesidad de repensar de manera holística los conceptos de vulnerabilidad y riesgo: una crítica y una revisión necesaria para la gestión. En Conference of Vulnerability in Disaster Theory and Practice, para International Work.

Cisneros, J. L. (2014). Niños y jóvenes sicarios: una batalla cruzada por la pobreza. El Cotidiano. Recuperado de: http://www.redalyc.org/articulo. oa?id=32531428002

CONEVAL. (2017). CONEVAL informa la evolución de la pobreza 20102016. Recuperado de http://www.coneval.org.mx/SalaPrensa/ Comunicadosprensa/Documents / Comunicado-o9-Medicionpobreza-2016.pdf

Convención Internacional sobre los Derechos de las Personas con Discapacidad. (2006). Recuperado de www.un.org/esa/socdev/enable/documents/ tccconvs.pdf 
Cordero D. (2012). Los 3 programas sociales que pule Enrique Peña Nieto Combate al hambre. UN1ÓN. Recuperado de http://archivo.unionguanajuato.mx/ articulo/2012/12/o2/gobierno/guanajuato/los-3-programas-socialesque-pule-enrique-pena-nieto

Diario Oficial de la Federación. (2014). Artículo 4. Recuperado de http://www. dof.gob.mx/nota_detalle.php?codigo $=5332025 \&$ fecha $=10 / 02 / 2014$

Discapacidad y Derechos. (2012). Recuperado de: https://www.youtube.com/ watch? $\mathrm{v}=1 \mathrm{gg} 097 \mathrm{y} 5 \mathrm{bHw}$

Fletes, R. (2004). Asistencia social: alcances y limitaciones. Revista de Estudios Jaliscienses (55).

Gobierno de la Federal. (2014). Programa Nacional de Asistencia Social 20142018, México.

Gobierno Federal. (2016). Catálogo de Programas Federales para Municipios 2016. Recuperado de: https://www.gob.mx/cms/uploads/attachment/ file/212316/Catalogo_de_Programas_Federales_2016_vf.pdf

Gobierno Federal. (2017). Transparencia Presupuestaria 2017. Recuperado de http://www.transparenciapresupuestaria.gob.mx/

Gramsci, A. (1999). Los cuadernos de la cárcel. Tomo V. México: Benemérita Universidad Autónoma de Puebla.

Instituto Nacional de Estadística y Geografía [INEGI] (2016). Estadísticas a propósito del Día del niño (30 de abril). Recuperado de: http://www.inegi. org.mx/saladeprensa/aproposito/2016/niño2016_o.pdf

Instituto Nacional de Estadística y Geografía [INEGI] (2017). Estadísticas a propósito del... día mundial contra el trabajo infantil. Recuperado de http:// www.inegi.org.mx/saladeprensa/aproposito/2017/infantil2017_Nal.pdf

Ley de asistencia social. (2014). Diario Oficial de la Federación. Recuperado de http://www.diputados.gob.mx/LeyesBiblio/pdf/270_191214.pdf 
Murillo, S. (2007). Naturalización de la pobreza y la desigualdad Efectos políticos y subjetivos de las estrategias del Banco Mundial. En Revista del Centro Cultural de la Cooperación (1).

Organización de las Naciones Unidas. (2006). Vigésimo tercer informe del Secretario General. Recuperado de: http://www.un.org/es/comun/ $\operatorname{docs} /$ ?symbol $=\mathrm{S} / 2006 / 948$

Paris, M. L. Zenteno, R., Treviño, J. y Wolf, S. (2015). Un análisis de los actores políticos y sociales en el diseño y la implementación de la política y la gestión migratoria en México, informe. Tijuana, México: El Colegio de la Frontera Norte.

Peña Nieto E. (2016a). Plan Nacional de Desarrollo 2013-2018. Recuperado de http://pnd.gob.mx

Peña Nieto, E. (2016b). El 4to Informe de Gobierno (2015- 2016). Recuperado de http://www.gob.mx/presidencia/articulos/4to-informe-degobierno-62351

PRI. (2012). Campaña. Presentó Enrique Peña Nieto Sus Propuestas Para Un México Mejor. Recuperado de: http://pri.org.mx/SomosPRI/ Saladeprensa/Nota.aspx?y=7009

Radio Fórmula. (2012). Mensaje íntegro de inicio campaña Enrique Peña Nieto. Radio Fórmula. Recuperado de http://www.radioformula.com.mx/notas. asp? Idn $=234628$

Ramírez, M. (2011). Política social, identidad y ciudadanía: La asistencia social vista por quienes la reciben. Identidades sociales y ciudadanía, 5(10), 137165.

Save The Children. (2017). Los peligros para la niñez. Indicadores para México. Estados Unidos. Save the Children.

SNDIF (2015). Meta, cero hambre. Recuperado de https://www.gob.mx/ difnacional\#prensa 
SNDIF (2013). Inaugura Angélica Rivera de Peña los XL Juegos Nacionales deportivos sobre sillas de ruedas. Recuperado de https://www.gob.mx/ difnacional\#prensa

SNDIF (2013). DIF Nacional se suma a medición de alimentación y desnutrición. Recuperado de https://www.gob.mx/difnacional\#prensa

SNDIF (2013). DIF nacional crea condiciones de equidad para personas con discapacidad: Angélica Rivera. Recuperado de https://www.gob.mx/ difnacional\#prensa

SNDIF (2013). Entrega Presidenta del DIF apoyos funcionales. Recuperado de https://www.gob.mx/difnacional\#prensa

SNDIF (2013). Posada intergeneracional en casa hogar para niñas. Recuperado de https://www.gob.mx/difnacional\#prensa

SNDIF (2013). Reafirma DIF Nacional compromiso para ejecutar políticas de inclusión social para personas con discapacidad. Recuperado de https:// www.gob.mx/difnacional\#prensa

SNDIF (2013). Angélica Rivera Encabeza acciones por la discapacidad. Recuperado de https://www.gob.mx/difnacional\#prensa

SNDIF (2005). Informe de rendición de cuentas (Primera etapa). México, SNDIF.

SNDIF (2015). Consulta Nacional de Información Básica a los Sistemas Municipales para el Desarrollo Integral de la Familia 2015.

SNDIF. (2016). Lineamientos de la Estrategia Integral de Asistencia Social Alimentaria 2017. Recuperado de https://www.gob.mx/ difnacional\#prensa

SNDIF (2013). Angélica Rivera entrega mochilas y útiles escolares en el Estado de México. Recuperado de https://www.gob.mx/difnacional\#prensa

SNDIF (2014). Incorporan a la Cruzada Nacional contra el Hambre a mil 800 comedores Oaxaqueños. Recuperado de https://www.gob.mx/ difnacional\#prensa 
SNDIF (2013). Llama DIF a unir esfuerzos en favor de los migrantes. Recuperado de https://www.gob.mx/difnacional\#prensa

SNDIF (2013). Hemos reunido 680 toneladas de víveres y otros apoyos para damnificados: Angélica Rivera de Peña. Recuperado de https://www.gob. $\mathrm{mx} /$ difnacional\#prensa

SNDIF (2013). Refrenda DIF Nacional compromiso con la infancia y adolescencia migrante. Recuperado de https://www.gob.mx/difnacional\#prensa

SNDIF (2015). Atendidos más de cuatro millones de mexicanos en seis mil comedores comunitarios de todo el país. Recuperado de https://www.gob. $\mathrm{mx} /$ difnacional\#prensa

SNDIF (2014). Cerca de 7 mil personas atendidas por DIF, fueron beneficiados por Fundación Cinépolis. Recuperado de https://www.gob.mx/ difnacional\#prensa

SNDIF (2013). Celebra DIF Nacional día del niño en la casa hogar para niñas “Graciela Zubirán”. Recuperado de https://www.gob.mx/ difnacional\#prensa

SNDIF (2015). Convoca Angélica Rivera de Peña crear más fuentes de empleo para discapacitados. Recuperado de https://www.gob.mx/difnacional\#prensa

SNDIF (2013). El gobierno de la república y UNICEF suman esfuerzos para proteger derechos de infancia y adolescencia. Recuperado de https:// www.gob.mx/difnacional\#prensa

SNDIF (2013). DIF Nacional atiende a 2 millones de personas con discapacidad al año. Recuperado de https://www.gob.mx/difnacional\#prensa

SNDIF (2012). La inclusión de personas con discapacidad no es una dádiva. Recuperado de https://www.gob.mx/difnacional\#prensa

SNDIF (2013). Crean ustedes mismos para seguir adelante, dijo a niños Angélica Rivera de Peña. Recuperado de https://www.gob.mx/difnacional\#prensa 
SNDIF (2013). Gobierno de la república vela para que niñez migrante no acompañada ejerza derechos. Recuperado de https://www.gob.mx/ difnacional\#prensa

Talavera, A. (2004). Diagnóstico de las áreas, servicios y talleres del DIF de San Andrés Cholula: elementos para una mejor difusión interna y externa (Trabajo de pregrado). Universidad de las Américas Puebla, Cholula, Puebla, México.

Valckx, A. (2007). Más que "callejeros": discursos y prácticas en los programas del Sistema Municipal para el Desarrollo Integral de la Familia, Puebla y la Fundación Junto con las Niñas y los Niños (JUCONI) (Trabajo de pregrado). Universidad de las Américas Puebla, Cholula, Puebla. 